\title{
Preparation and Property Research of Strain Sensor Based on PDMS and Silver Nanomaterials
}

\author{
Lihua Liu, Qiang Zhang, Dong Zhao, Aoqun Jian, Jianlong Ji, Qianqian Duan, \\ Wendong Zhang, and Shengbo Sang
}

\author{
MicroNano System Research Center, Key Laboratory of Advanced Transducers and Intelligent Control System, \\ Ministry of Education and Shanxi Province and College of Information Engineering, Taiyuan University of Technology, \\ Taiyuan 030024, China
}

Correspondence should be addressed to Shengbo Sang; sangshengbo@tyut.edu.cn

Received 10 January 2017; Accepted 20 February 2017; Published 9 March 2017

Academic Editor: Kourosh Kalantar-Zadeh

Copyright (C) 2017 Lihua Liu et al. This is an open access article distributed under the Creative Commons Attribution License, which permits unrestricted use, distribution, and reproduction in any medium, provided the original work is properly cited.

\begin{abstract}
Based on the advantages and broad applications of stretchable strain sensors, this study reports a simple method to fabricate a highly sensitive strain sensor with Ag nanomaterials-polydimethylsiloxane (AgNMs-PDMS) to create a synergic conductive network and a sandwich-structure. Three $\mathrm{Ag}$ nanomaterial samples were synthesized by controlling the concentrations of the $\mathrm{FeCl}_{3}$ solution and reaction time via the heat polyols thermal method. The AgNMs network's elastomer nanocomposite-based strain sensors show strong piezoresistivity with a high gauge factor of 547.8 and stretchability from $0.81 \%$ to $7.26 \%$. The application of our high-performance strain sensors was demonstrated by the inducting finger of the motion detection. These highly sensitive sensors conform to the current trends of flexible electronics and have prospects for broad application.
\end{abstract}

\section{Introduction}

In recent years, along with the progression of information science, materials science, electronic technology, and micro/ nanotechnology, flexible electronic technology is rapidly emerging as a new industry. Due to the development of film materials and technology, flexible electronic technology has been applied to plasma display panels [1], liquid crystal displays, organic laser displays [2-4], solar cells [5], optical amplifiers [6], and electric pulse displays and transparent tablets [7]. Flexible, scalable, and wearable electronic devices can be easily installed in the clothing or directly connected to the body, designed for the flexibility of human movement with sensitive strain sensors.

Flexible electronic skin (e-skin) is a popular application in the field of flexible electronics. E-skins are already capable of providing augmented performance over their organic counterpart, both in superior spatial resolution and in thermal sensitivity [8]. Throughout the development of eskin, it has gained the capability of monitoring and sensing stimuli and can be used for various applications [9], including robots $[10,11]$, human-machine interfaces $[12,13]$, touch detection [14, 15], strain gauges [16], temperature monitoring [17], and medicine and healthcare [18, 19]. Strain sensors are fundamental components of e-skin devices, and the preparation of highly sensitive large-area strain sensors is critical to the development of e-skins [20]. Piezopotentialpowered sensor devices [21] for use in e-skin and wearable applications are particularly desirable-or even required for certain biomedical implants [22].

A flexible base is an important part of flexible electronics, not only because it provides flexible support but also because it functions as a material, which can produce, transfer, and process mechanical and electrical signals.

Due to the fact that PDMS has good transmission to light, good biocompatibility, and the structure of low Young's modulus and a variety of physical and chemical properties, it is one of the unique materials for microimaging and microfluidic channeling. And its high flexibility and repeatability make PDMS a good transparent rubber polymer as applied to the fields of flexible devices [23-25]. King and others used sugar stents in noncuring polydimethylsiloxane contained carbon black and generated a new type of material [26]. Jung and others in the University of South Korea fabricated the 
electrocardioelectrode based on CNTs-PDMS materials and compared how different concentrations of carbon nanotubes and different electrode diameter affect the stability of the electrocardiogram signal [27]. Yi and others presented a solution for enhancing the thermal conductivity of such PDMS based microfluidics by introducing thermally conductive alumina nanoparticles [28]. Moreover, due to the fact that PDMS itself has no electrical conductivity, nanomaterials can be embedded into structural materials and operate as both multidirectional and multifunctional sensors with high strain resolution and outstanding electrical properties [27].

In this study, we study highly flexible, sensitive, and reliable strain sensors based on the sandwich-structure (i.e., silver nanomaterials embedded between two layers of PDMS). First, we controlled different concentrations of $\mathrm{FeCl}_{3}$ solution at different reaction times to obtain and research three different productions. Second, we observed the morphology of products through SEM and analyzed the main elements in the products by EDS. Third, based on PDMS as a flexible base, we made a model by filling the different products between the two layers of PDMS and then received three simple strain-sensing units. The strain-sensing characteristics of the sensors, including stretch response under static and dynamic loads, stretchability, and sensitivity, have been investigated. After analyzing the different sensors' test results, our strain sensors showed superior sensitivity (especially in small variables) over conventional sensors. The unique properties of these strain gauges have the potential to improve existing technology and reveal new fields of application. The results of this experiment can provide support for the development of wearable devices. At the same time, the sensor technology is simple, has lower equipment requirements, has lower production costs, and is available in various daily flexible electronic devices.

\section{Materials and Methods}

2.1. Synthesis of Silver Nanowires (AgNWs) and Nanoparticles (AgNPs). AgNWs and AgNPs were synthesized by a modified polyol method. Ethylene glycol $(100 \mathrm{~mL})$ was heated at $160^{\circ} \mathrm{C}$ for $1 \mathrm{~h}$ with a magnetic stirrer (stirring speed $=$ $250 \mathrm{rpm}$ ). A certain concentration of $\mathrm{FeCl}_{3}$ in ethylene glycol as the growth control agent was added to the ethylene glycol that was heated afterwards. After the new solution was stirred evenly for another $15 \mathrm{~min}, 5 \mathrm{~mL}$ of $3.6 \mathrm{M}$ polyvinylpyrrolidone (PVP) in ethylene glycol was added to the system. Then, $5 \mathrm{~mL}$ of $0.6 \mathrm{M} \mathrm{AgNO}_{3}$ in ethylene glycol was injected drop by drop into the solution. After the $\mathrm{AgNO}_{3}$ solution was injected, the solution was heated for a period of time and placed into an ice bath to stop the reaction. After the solution was cooled, a large amount of anhydrous ethanol was added to the solution. The solution was centrifuged at $6000 \mathrm{rpm}$ for $5 \mathrm{~min}$ and washed three times with anhydrous ethanol to remove the excess PVP and ethylene glycol. AgNMs (Ag nanomaterials) were stored in isopropyl alcohol (IPA) for further experiments.

When the concentration of $\mathrm{FeCl}_{3}$ was $8 \mathrm{mmol} / \mathrm{L}$, reaction time of $40 \mathrm{~min}$, we received $\mathrm{AgNMs}$. When $\mathrm{FeCl}_{3}$ was $6 \mathrm{mmol} / \mathrm{L}$, reaction time for $38 \mathrm{~min}$, results were AgNMs2.

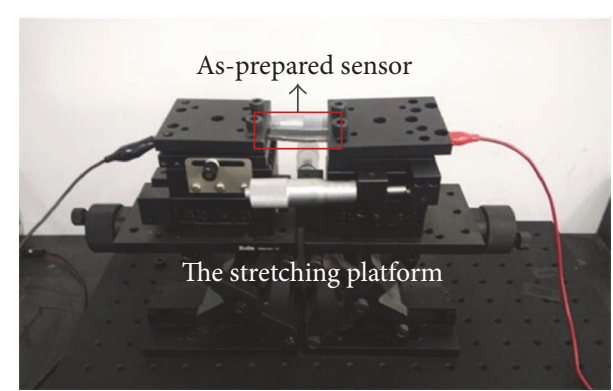

FIGURE 1: The sensor testing platform: the optical flat OTSB345-1. In the platform center is the as-prepared product.

When $\mathrm{FeCl}_{3}$ was $6 \mathrm{mmol} / \mathrm{L}$, reaction time for $58 \mathrm{~min}$, results were AgNMs3.

2.2. Fabrication of the Strain Sensors. The strain sensors were fabricated by the following procedure: the first step involved the fabrication of a PDMS mold. A mixture of PDMS monomer and curing agent with a ratio of $10: 1$ was degassed to remove any bubbles and poured into the center of a Petri dish (diameter: $90 \mathrm{~mm}$ ) placed in a glass mold $(1.5 \mathrm{~cm} * 5 \mathrm{~mm}$ $* 0.5 \mathrm{~mm}$ ). The Petri dish and its contents were cured at $75^{\circ} \mathrm{C}$ for 1 hour in an oven. After curing, the PDMS was carefully separated from the Petri dish and the glass mold, yielding the PDMS mold. The second step involved fabricating the AgNMs-PDMS strain sensors. Copper tape was pasted on both ends of groove in the mold intended to function as an electrode. The AgNMs1, AgNMs2, and AgNMs3 that were previously prepared were scattered in isopropyl alcohol and dropped, respectively, onto three PDMS molds. Three molds were placed half an hour in the normal temperature. After the solution dried and PDMS was poured into surface of the groove, the three molds were cured at normal temperature for $24 \mathrm{~h}$. The cured PDMS film in the center was cut into three rectangles to be used as samples. Based on AgNMs1, AgNMs2, and AgNMs3, we, respectively, created the products Sensor 1, Sensor 2, and Sensor 3.

2.3. Characterizations of Compositions and Morphologies of the Products. The morphology and structure of Ag nanomaterials (AgNMs) were observed by using scanning electron microscopy (SEM) images obtained by using SU3500 SEM (Hitachi Corporation) operated at $15 \mathrm{kV}$. Energy-dispersive $\mathrm{X}$-ray (EDX) analysis was also performed with the same instrument. Transmission electron microscopy (TEM) was carried out with a JEM-2010 FX instrument operating at $200 \mathrm{kV}$ accelerating voltage.

2.4. Sensor Test Platform. For the AgNMs-PDMS sensors tensile test, the experimental platform was built as shown in Figure 1. Composite film was placed in the optical flat OTSB345-1, with a three-dimensional displacement on the stage, clamped to adjust to the same plane, and the apparatus was clamped tight to adjust to the spiral rod indentation and promote composite film stretching. After being connected to the ends of the composite film electrodes and Keithley2400 of measure instruments, the film was tested for the resistance of 


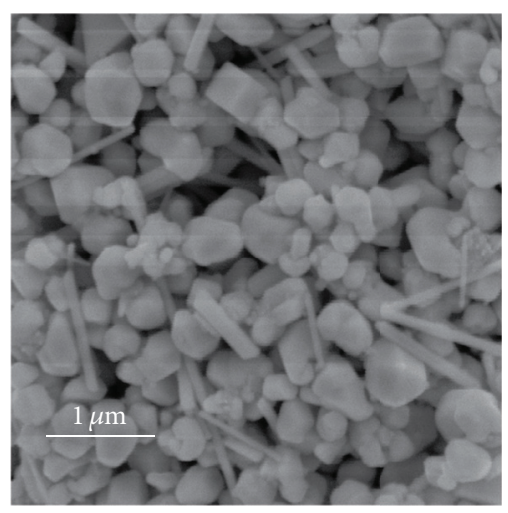

(a)

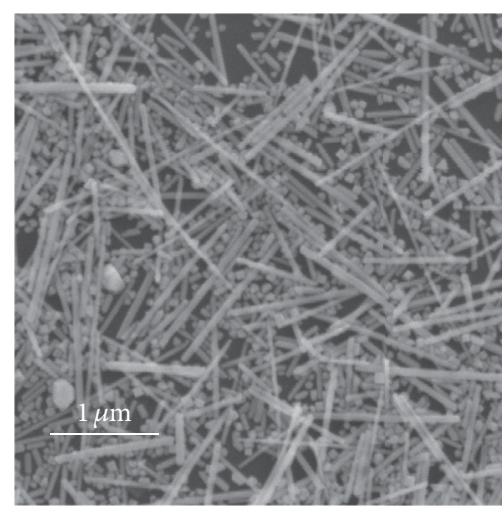

(b)

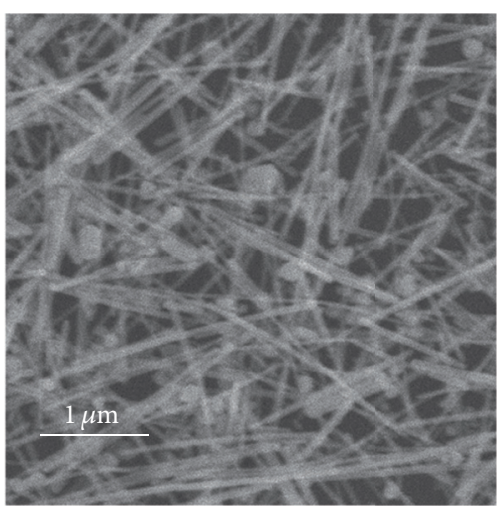

(c)

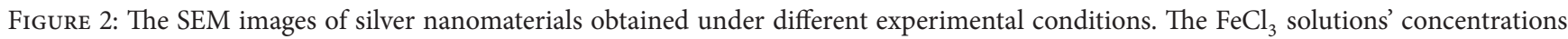
and the oil bath time are (a) $8 \mathrm{mmol} / \mathrm{L}$ and $40 \mathrm{~min}$, (b) $6 \mathrm{mmol} / \mathrm{L}$ and $38 \mathrm{~min}$, and (c) $6 \mathrm{mmol} / \mathrm{L}$ and $58 \mathrm{~min}$, respectively.

the original length. Then the screw rod was rotated in half of a circle repeatedly (elongation $0.25 \mathrm{~mm}$ ) to measure the composite film resistance until the resistance of the composite membrane became infinite.

A Keithley 2400 source unit of measure instrument was used to test the films created by our experiment methods. The films were tested with RS232 USB serial port connection and computer software of the Keithley2400. Results showed the output data and the origin software to analyze the data.

\section{Results and Discussion}

As compared with most reported strain sensors fabricated by depositing or embedding the sensing materials on the flexible substrates (in which the structure and performance of the strain sensors can be easily damaged even by mild touching) $[29,30]$, our sandwich-structured strain sensor can easily be handled by complete encapsulation. The sensors can be mounted directly on the skin and easily attached to complex surfaces without causing any damage to the nanocomposite thin film.

Our study tested whether differences existed in the synthesis of silver nanowires by applying $\mathrm{FeCl}_{3}$ with varying concentrations. In addition, varying reaction times were employed to compare the synthesis process. In Figure 2, three images with different proportions of AgNMs products composed of AgNWs and AgNPs are pictured. As shown in Figure 2(a), the SEM shows that when the concentration of $\mathrm{FeCl}_{3}$ was $8 \mathrm{mmol} / \mathrm{L}$ and the reaction time was $40 \mathrm{~min}$, the synthesis of silver nanowires failed and the majority of the structures were silver nanoparticles in Product 1 . With the $6 \mathrm{mmol} / \mathrm{L} \mathrm{FeCl}$, with $38 \mathrm{~min}$ of reaction time, these large silver nanoparticles were able to grow into rod-shaped structures as shown in Figure 2(b). The synthesized silver nanostructures still contained a large amount of AgNPs and AgNWs. Note that some silver particles did not develop into the rod-shaped structures and kept growing into smaller colloids that were as stable as the wires. All steps were the same as above in the synthesis of the silver materials, except the fact that the reaction time increased to $58 \mathrm{~min}$,

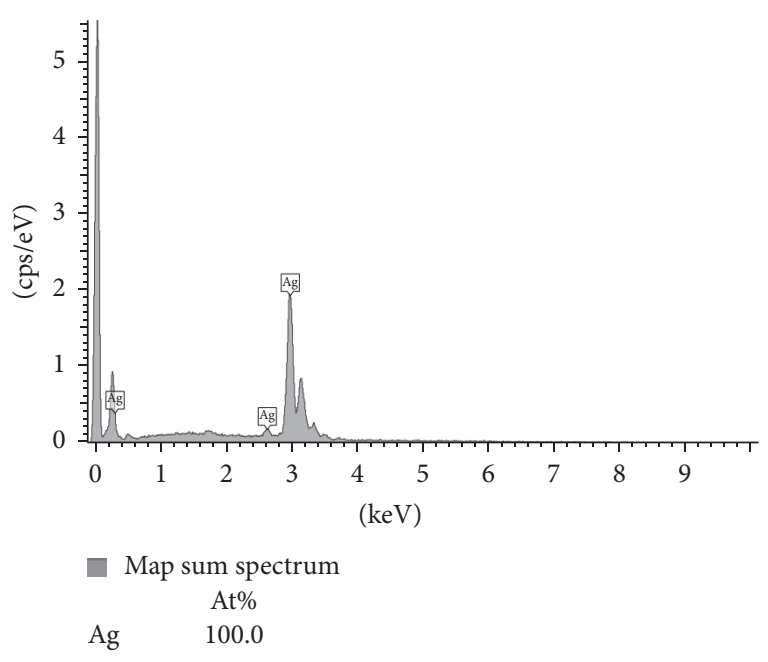

FIGURE 3: The energy-dispersive X-ray spectrum (EDS) of the silver nanomaterials.

as shown by the SEM image of Product 3 in Figure 2(c). In Product 3 these particles could coexist in the solution with the silver wires. The silver nanowires demonstrated an average length of $6 \mu \mathrm{m}$ and an average width of $62 \mathrm{~nm}$. The results suggest that, by adding $\mathrm{FeCl}_{3}$ in an appropriate concentration and by changing the reaction time, the synthesis of silver nanomaterials can effectively be achieved.

The chemical composition of AgNMs was obtained using energy-dispersive X-ray spectroscopy (EDS) during SEM. The EDS characterizations of AgNMs on silicon substrate are showed in Figure 3. The results show that the product of $\mathrm{Ag}$ content under the condition of all three experiments was $100 \%$, excluding other substances.

The cross-sectional optical images of the sensors are shown in Figure 4 indicating well-patterned AgNMs-PDMS nanocomposite strain sensors. In Figure 4(a), the SEM images are on the transverse section of Sensor 3, with an average thickness of $6.5 \mu \mathrm{m}$, showing the transverse section figure of neat rows of AgNWs and small amounts of AgNPs. 


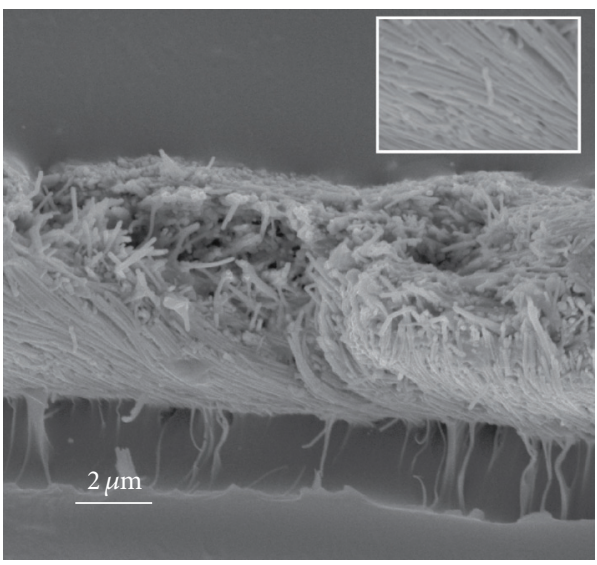

(a)

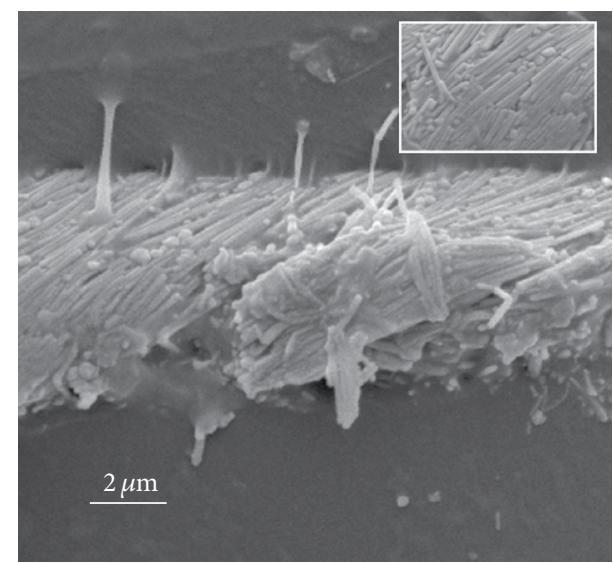

(b)

FIGURE 4: Optical microscope images on cross-section of the sandwich-structure strain sensor: Sensor 2 (a); Sensor 3 (b). The insert, respectively, shows the local amplification in (a) and (b).

Figure 4(b) demonstrates the cross-sectional SEM image of the sandwich-structured Sensor 2, with the average thickness of $5.7 \mu \mathrm{m}$. There are a lot of AgNWs and AgNPs mixed and arranged in a more randomized fashion. The insert, respectively, shows the local amplification in Figures 4(a) and 4(b). When the liquid PDMS is cast onto the AgNMs film, the liquid PDMS penetrates the interconnected pores of the three-dimensional AgNMs network, owing to the low viscosity and low surface energy of the liquid PDMS. After curing the PDMS, all silver nanomaterials are buried into the PDMS surface without significant voids, showing good adhesion between the AgNMs and PDMS substrate. The liquid PDMS completely penetrated the AgNMs network thin film on two sides and filled the gaps between nanomaterials, forming a robust nanocomposite of AgNMs and PDMS.

Using Keithley2400 testing software, we scanned the voltage range from $-1 \mathrm{~V}$ to $1 \mathrm{~V}$ and measured $I-V$ curve of thin films at room temperature. As shown in Figure 5, curve A shows $I-V$ of Sensor 1 consisted mostly of AgNPs and $I-V$ of Sensor 2 consisted of a fair amount of AgNPs and AgNWs (as curve B), while curve C shows that $I-V$ of Sensor 3 consisted primarily of AgNWs. Through analysis, the resistance of Sensor 1 was $100 \Omega$. When the sensor showed a large number of nanoparticles, Ag nanomaterials isolated existence and the resistance value was higher. The resistance of Sensor 2 was $16.67 \Omega$, and there was a certain proportion of composite silver nanomaterials, which easily formed the conductive path-therefore, the more paths, the higher the possibility of the sensor having good conductivity and a lower resistance. The resistance based on Sensor 3 was $33.33 \Omega$, which consisted of more nanowires and fewer nanoparticles. The sensor did not easily form the conductive path. The easier it is to form a connected network, the less resistance there will be. Synergy between AgNWs and AgNPs ensured that the sensor had good electrical conductivity and resulted in small resistance at the unstretched state.

Sensor 1 is open circuit as soon as stretching occurs. The AgNMs1 were connected and Sensor 1 was conductive when there was no deformation on Sensors 1. Relative large amount

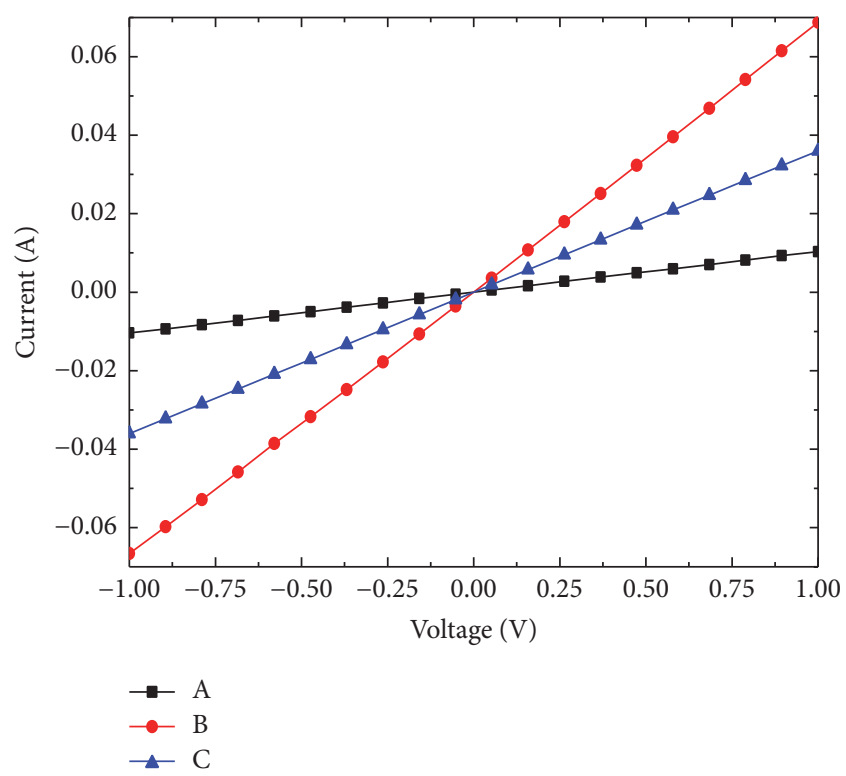

Figure 5: Current-voltage curves of three different AgNMs-PDMS strain sensors at zero tensile. (A) $I-V$ of Sensor 1 consisted mostly of AgNPs. (B) $I-V$ of Sensor 2 consisted of a fair amount of AgNPs and AgNWs. (C) $I-V$ of Sensor 3 consisted primarily of AgNWs.

of Ag nanowires still forms a conductive network during deformation. However, the AgNMs1 were separated and the electrons path did not exist during stretching because of the small amount of Ag nanowires.

The results of AgNMs composite film tensile test analysis were as follows: after different stretching, the spatial structure of silver nanoparticles and nanometer silver wire was changed. As shown in Figure 6(a), Sensor 2 stretched from $0.81 \%$ to $11.34 \%$, and the resistance variation was as high as 31.52. To investigate the application of these strain sensors, the gauge factor (GF) was adopted to measure the data for our strain sensor under variable strains, which is the standard for quantifying the piezoresistive sensitivity. It is determined on 


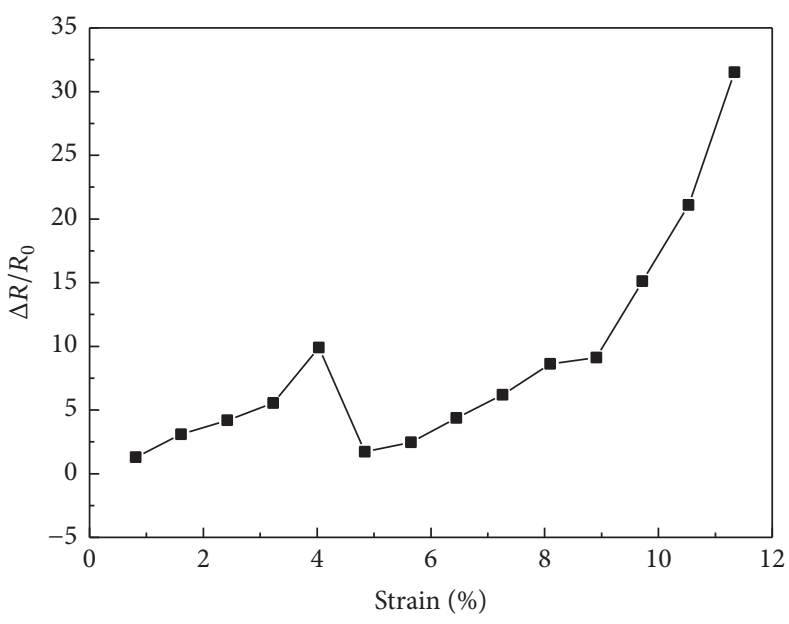

- - Sensor 2

(a)

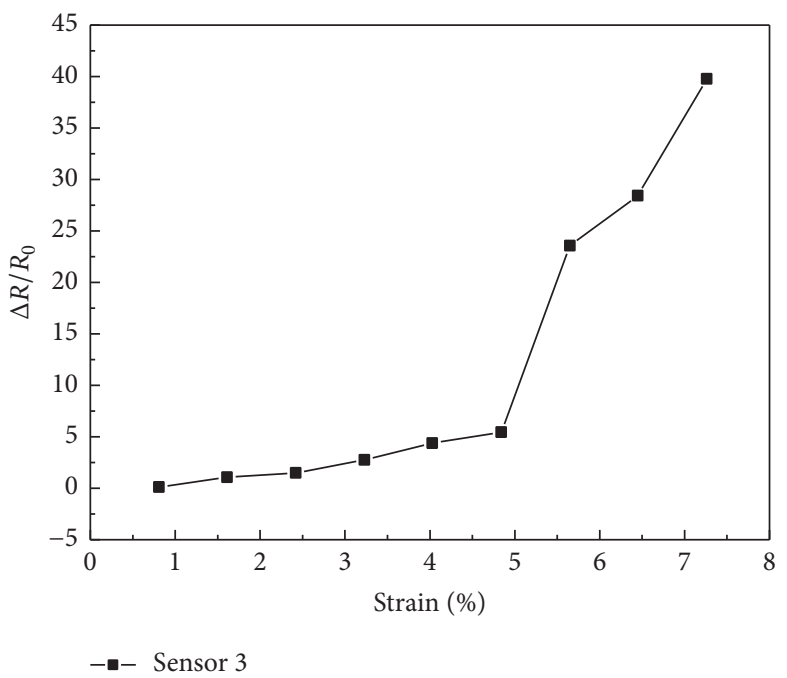

(c)

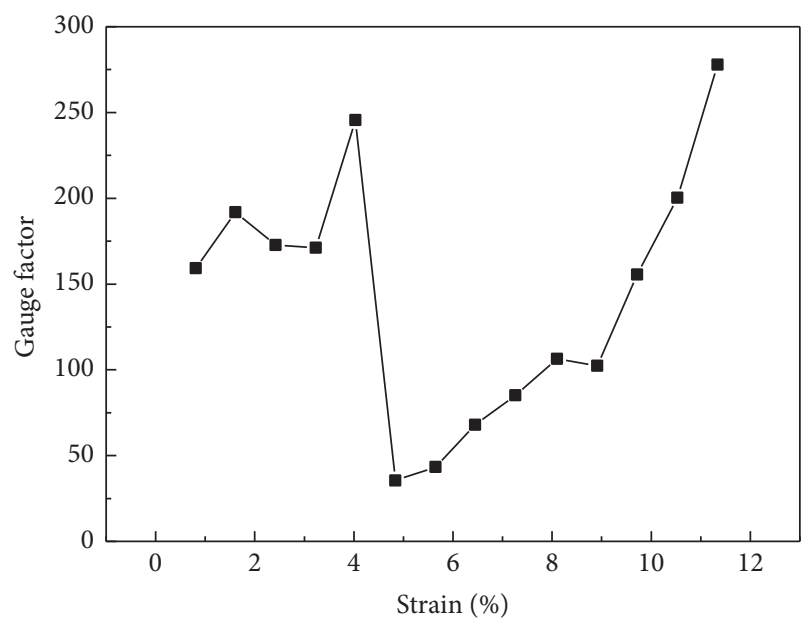

- - Sensor 2

(b)

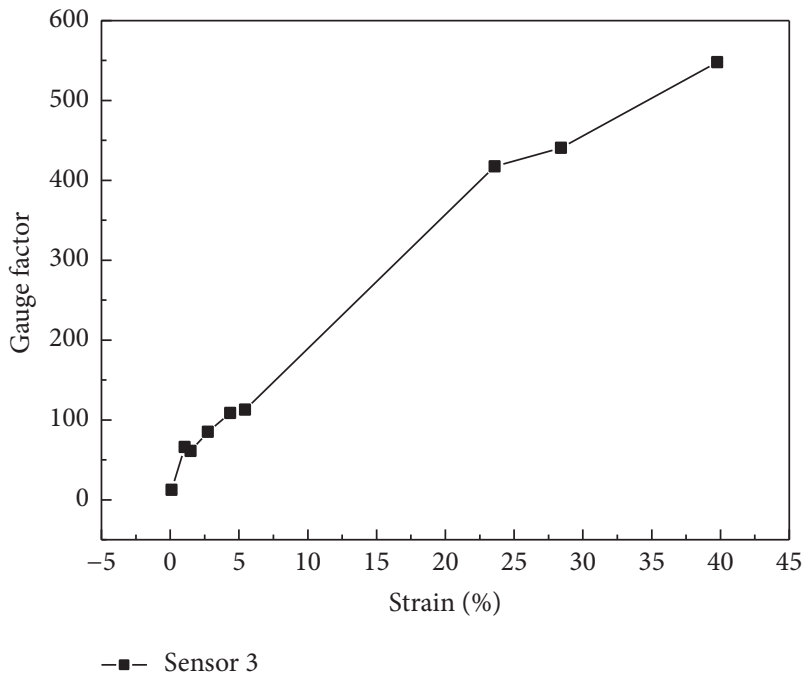

(d)

FIGURE 6: Relative change of resistance versus strain for Sensor 2 (a) and Sensor 3 (c). Gauge factor under variable strain of Sensor 2 (b) and Sensor $3(d)$.

the basis of elongation experiments, by using the following equation:

$$
\mathrm{GF}=\frac{\Delta R / R_{0}}{\Delta L / L_{0}}=\frac{\Delta R}{\varepsilon R_{0}} .
$$

Here, $\Delta R$ and $\Delta L$ are the changes in the electrical resistance and the elongation length, respectively $\left(\Delta R=\left|R-R_{0}\right|, \Delta L=\right.$ $\left.\left|L-L_{0}\right|\right) . R_{0}$ and $L_{0}$ are the initial values of the resistance and the length, respectively. The GF is also affected by the composition of silver nanomaterials. In Figure 6(a), it shows that the resistance change is unpredictable and repeatability is not good-even $\Delta R / R_{0}$ happened to jump under the small deformation range. In Figure 6(b), the GF showed a trend of instability. The analysis of reasons is as follows: while starting to stretch, AgNWs2 (due to the mutual connection between particles and the particles being less than the cut-off distance of tunneling effect), the composite film ends up having good conductivity. When the composite film was stretched, there appeared to be a downward trend. After stretching to a certain extent, AgNWs are unable to connect to each other, the original conductive network gets destroyed, and a new conductive network connecting the path through the AgNPs creates a tunneling effect. Due to the change of the internal structure, the variables changed are uncertain.

As shown in Figure 6(c), Sensor 3 is stretching from $0.81 \%$ to $7.26 \%$; resistance variation is as high as 39.77 . Figure $6(\mathrm{~d})$ illustrates the GF of the strain sensor as a function of strain. It shows, for various tension degrees, that the highest value of GF is 547.8 for $7.26 \%$ tension and the lowest value is 12.3 for $0.81 \%$ tension of Sensor 3. Compared with other reported stretchable strain sensors, Sensor 3 has a high GF and good electrical conductivity prior to stretching. Sensor 3 had large amounts of AgNWs, and, due to this fact, the conductive interface could consist of the interface between the AgNWs and AgNPs. The interface between them is divided into 


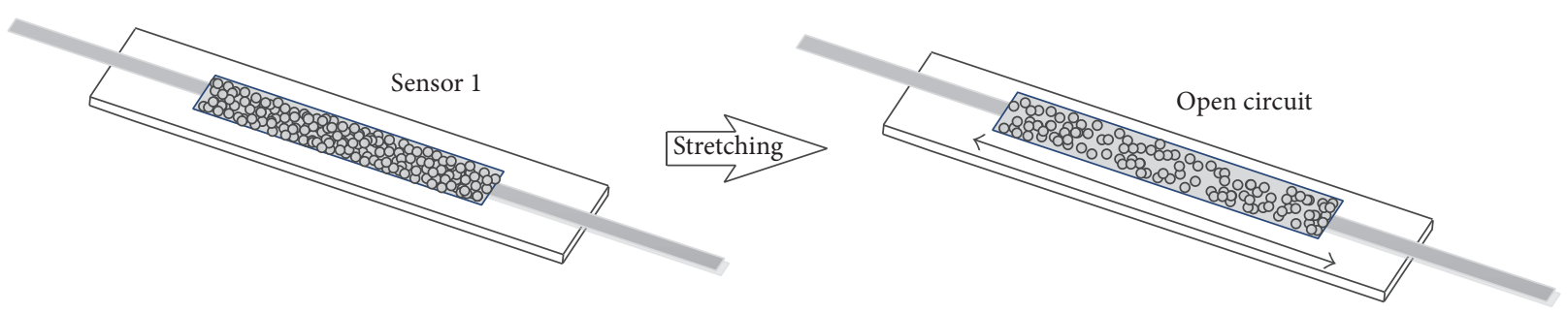

(a)

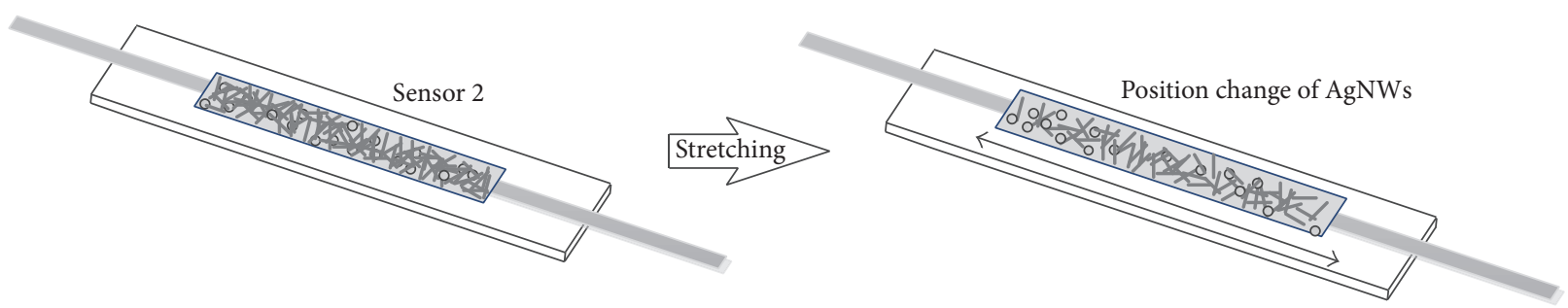

(b)

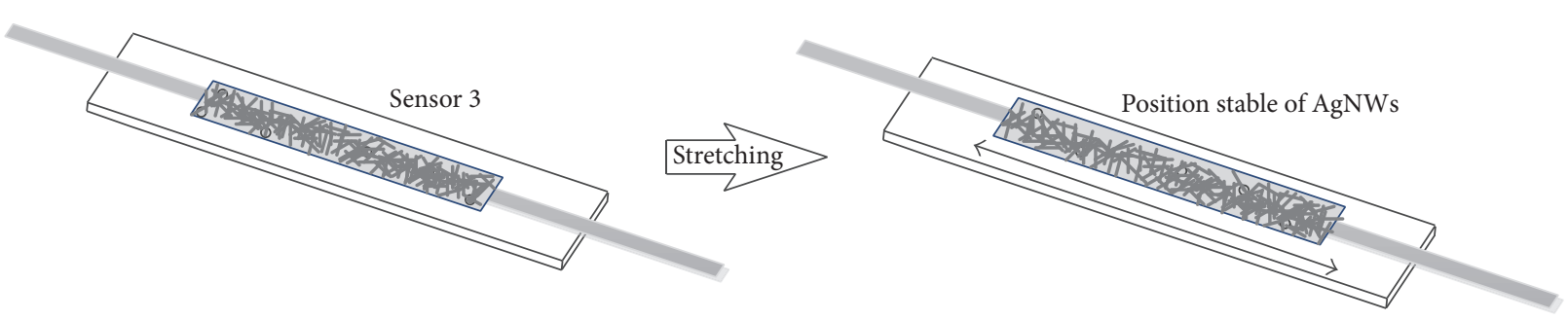

(c)

FIgURE 7: Schematic sensing model of Sensor 1, Sensor 2, and Sensor 3.

three categories: no contact resistance (fully contact), having a certain cut-off distance of tunnel junction, or complete disconnection.

To understand the resistance variations of Sensor 1, Sensor 2, and Sensor 3 in the stretching process, we propose a simple model to describe the working principle of sensors, as shown in Figure 7. As shown in Figure 7(a), the lager amounts of $\mathrm{Ag}$ nanoparticles in Sensor 1 were connected and Sensor 1 was conductive at the original condition. However, the electrons path was destroyed during stretching. Compared with Sensor 1, sensitive unit in Sensor 2 contains more AgNWs, which is shown as Figure 7(b). Due to the existence of AgNPs, the internal structure changes during the stretching process are unstable, resulting in unstable resistance changes. The sensitive unit in Sensor 3 is composed of a large amount of silver nanowires in Figure 7(c). During the stretching, the position of the silver nanowires in unit is more stable than Sensor 1 and Sensor 2. Comparing Sensor 2 with Sensor 3, networks that consisted of AgNWs and AgNPs are not stable during stretching because the amount of AgNPs in Sensor 2 is higher. Due to the mutual connection between particles and the particles being less than the cut-off distance of the tunneling effect, AgNWs make the composite film have a good electrical conductivity, the tunneling resistance increases, $\Delta R$ is larger, and the strain sensor GF is greater.

When comparing Sensor 2 and Sensor 3, we came to the conclusion that Sensor 2 has better tensile performance than
Sensor 3, but Sensor 2 has different sensitivity and unstable resistance change. In the process of relative resistance variation by gradually increasing strain from $0.81 \%$ to $7.26 \%$, in comparison to Sensor 2, Sensor 3 had higher stability and GF. Especially in the small scope of deformation measurement, Sensor 3 AgNPs were relatively less compared to Sensor 2. Conductive path is hard to change, so Sensor 3 had better stability and sensitivity. The experiment was repeated four times to test the repeatability of Sensor 2 and Sensor 3. As shown in Figure 8(a), the test result was different almost every time, while the composite films of AgNMs2 responded to being stretched four times. This phenomenon shows that, in the process of stretching, the distance between particles is uncertain, therefore the resistance change is unpredictable, repeatability is not good, and even $\Delta R / R_{0}$ happened to jump under the small deformation range. However, in Figure 8(b), Sensor 3 responded to the stretch similarly all four times to establish the mathematical model for the trend of change of resistance. It showed good stability because when less AgNPs are perfectly embedded in network of AgNWs it is easy to form stable conductive pathways. Sensor 3's repeatability is better than Sensor 2 and therefore has more application value.

Our sandwich-structured AgNMs-PDMS strain sensor was used for a finger in the experimental process to detect a human finger's motion. Figure 9 shows the experimental process used for one finger's motion recognition. Solid line $\mathrm{A}$ and dotted lines $\mathrm{B}$, respectively, represent the different 


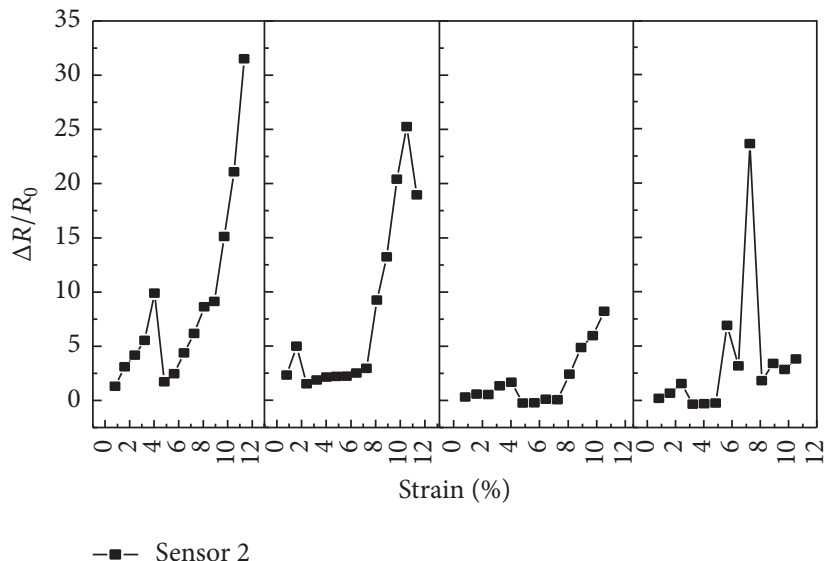

(a)

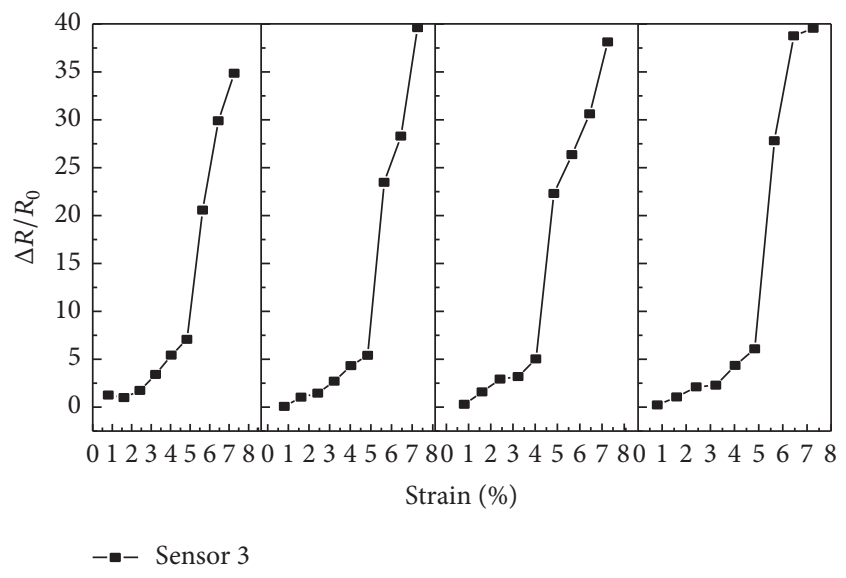

(b)

FIGURE 8: Relative change of resistance versus strain for the sensor to stretch four-times-repeated experiments. Sensor 2 (a); Sensor 3 (b).

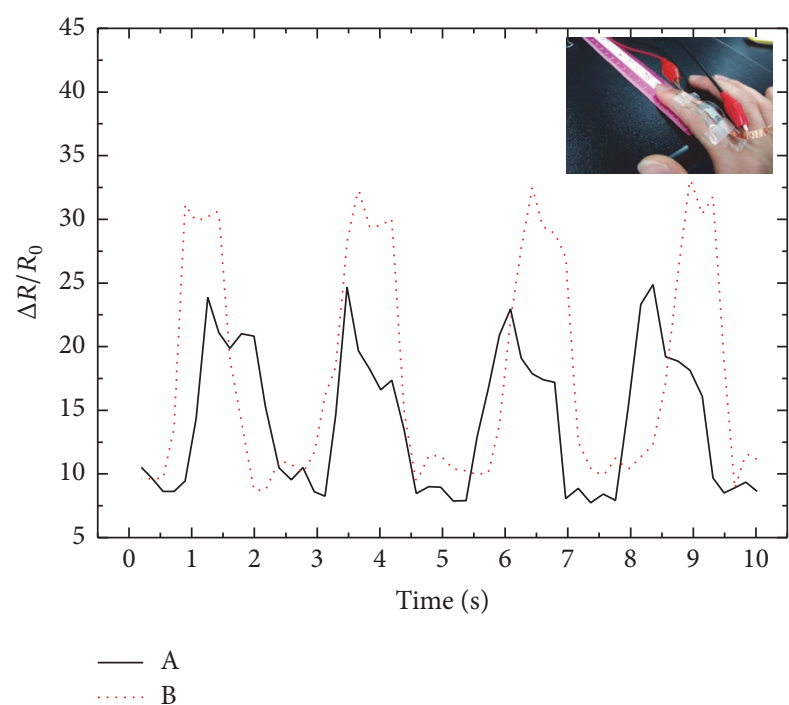

FIGURE 9: The experimental process used for one finger's motion recognition. Solid line A and dotted lines B, respectively, represent the different response results of the resistance change when a finger moves $1 \mathrm{~cm}$ and $2 \mathrm{~cm}$ in a horizontal direction.

response results of the resistance change when a finger moves $1 \mathrm{~cm}$ and $2 \mathrm{~cm}$ in a horizontal direction. By contrast, we can see four stretching-releasing cycles, respectively, and then the resistance variation to the strain sensor was increased when the process of stretching but the resistance variation decreased during the process of releasing. From this figure, we can get conclusion that when the finger moved in a horizontal displacement of the fixed distance and bent at a similar level, the peak position changed little, and the resistance variation stayed roughly the same and showed good stability. In comparison with the two different response curves of displacement, the curve of the horizontal displacement of $2 \mathrm{~cm}$ peak position was greater than $1 \mathrm{~cm}$. In a certain range, the larger the horizontal displacement, the better the tensile effect. From the application of the fingers' stretchingreleasing cycle results, it was observed that the strain sensor we prepared can come in direct contact with the skin and be used for monitoring human actions.

\section{Conclusion}

In summary, we developed a new type of highly sensitive, stable strain sensors with a low cost fabrication process based on the sandwich-structured AgNMs-PDMS nanocomposite. In the article, we first prepared different proportions of silver nanomaterials and based experiments on three different kinds of products for three kinds of strain sensors and tested the different sensors' performance. The tensile experiments results showed Sensor 3 to have adequate proportions of silver nanowires and particle composite membrane that can be stretched from $0.81 \%$ to $7.26 \%$ with the highest value of GF being 547.8 for $7.26 \%$ tension. The strain sensors with high GF obtained in this work conformed to the trend of the developing flexible electronics, having broad application prospects.

\section{Authors' Contributions}

Lihua Liu and Qiang Zhang contributed equally to this work.

\section{Conflicts of Interest}

The authors declare that there are no conflicts of interest regarding the publication of this paper.

\section{Acknowledgments}

This study was financially supported by the National Natural Science Foundation of China (nos. 61471255, 61474079, 61501316, 51505324, and 51622507), the Basic Research Program of Shanxi for Youths (nos. 2014021023-3 and 2015021092), the Research Project Supported by Shanxi Scholarship Council of China (2015-047), Excellent Talents Technology Innovation Program of Shanxi Province of China (201605D211023), and 863 Project (2015AA042601). 


\section{References}

[1] M. Fahland, P. Karlsson, and C. Charton, "Low resisitivity transparent electrodes for displays on polymer substrates," Thin Solid Films, vol. 392, no. 2, pp. 334-337, 2001.

[2] J.-I. Nomoto, J.-I. Oda, T. Miyata, and T. Minami, "Effect of inserting a buffer layer on the characteristics of transparent conducting impurity-doped $\mathrm{ZnO}$ thin films prepared by $\mathrm{dc}$ magnetron sputtering," Thin Solid Films, vol. 519, no. 5, pp. 15871593, 2010.

[3] D.-H. Kim, M.-R. Park, and G.-H. Lee, "Preparation of high quality ITO films on a plastic substrate using RF magnetron sputtering," Surface \& Coatings Technology, vol. 201, no. 3-4, pp. 927-931, 2006.

[4] U. Betz, M. K. Olsson, J. Marthy, M. F. Escolá, and F. Atamny, "Thin films engineering of indium tin oxide: large area flat panel displays application," Surface \& Coatings Technology, vol. 200, no. 20-21, pp. 5751-5759, 2006.

[5] F. C. Krebs, J. Fyenbo, and M. Jørgensen, "Product integration of compact roll-to-roll processed polymer solar cell modules: methods and manufacture using flexographic printing, slotdie coating and rotary screen printing," Journal of Materials Chemistry, vol. 20, no. 41, pp. 8994-9001, 2010.

[6] D. K. Kim, Y. Lai, B. T. Diroll, C. B. Murray, and C. R. Kagan, "Flexible and low-voltage integrated circuits constructed from high-performance nanocrystal transistors," Nature Communications, vol. 3, article 1216, 2012.

[7] A. C. Hsu, S. P. Day, R. Schediwy, and D. Gillespie, "Flexible transparent touch sensing system for electronic devices," United States Patent 7030860.

[8] M. L. Hammock, A. Chortos, B. C.-K. Tee, J. B.-H. Tok, and Z. Bao, "25th Anniversary article: the evolution of electronic skin (E-Skin): a brief history, design considerations, and recent progress," Advanced Materials, vol. 25, no. 42, pp. 5997-6038, 2013.

[9] Y. Huang, D. Fang, C. Wu, W. Wang, X. Guo, and P. Liu, "A flexible touch-pressure sensor array with wireless transmission system for robotic skin," Review of Scientific Instruments, vol. 87, no. 6, Article ID 065007, 2016.

[10] R. S. Dahiya, P. Mittendorfer, M. Valle, G. Cheng, and V. J. Lumelsky, "Directions toward effective utilization of tactile skin: a review," IEEE Sensors Journal, vol. 13, no. 11, pp. 4121-4138, 2013.

[11] J.-C. Kim, K.-S. Kim, and S. Kim, "Note: a compact three-axis optical force/torque sensor using photo-interrupters," Review of Scientific Instruments, vol. 84, Article ID 126109, 2013.

[12] X. Wang, H. Zhang, R. Yu et al., "Dynamic pressure mapping of personalized handwriting by a flexible sensor matrix based on the mechanoluminescence process," Advanced Materials, vol. 27, no. 14, pp. 2324-2331, 2015.

[13] X. Zhao, Q. Hua, R. Yu, Y. Zhang, and C. Pan, "Flexible, stretchable and wearable multifunctional sensor array as artificial electronic skin for static and dynamic strain mapping," Advanced Electronic Materials, vol. 1, no. 7, Article ID 1500142, 2015.

[14] X. Lee, T. Yang, X. Li et al., "Flexible graphene woven fabrics for touch sensing," Applied Physics Letters, vol. 102, no. 16, Article ID 163117, 2013.

[15] B. C.-K. Tee, A. Chortos, A. Berndt et al., "A skin-inspired organic digital mechanoreceptor," Science, vol. 350, no. 6258, pp. 313-316, 2015.
[16] N. Lu, C. Lu, S. Yang, and J. Rogers, "Highly sensitive skinmountable strain gauges based entirely on elastomers," Advanced Functional Materials, vol. 22, no. 19, pp. 4044-4050, 2012.

[17] R. C. Webb, A. P. Bonifas, A. Behnaz et al., "Ultrathin conformal devices for precise and continuous thermal characterization of human skin," Nature Materials, vol. 12, no. 10, pp. 938-944, 2013.

[18] K. Fukuda, Y. Takeda, Y. Yoshimura et al., "Fully-printed highperformance organic thin-film transistors and circuitry on onemicron-thick polymer films," Nature Communications, vol. 5, article 4147, 2014.

[19] M. Kaltenbrunner, T. Sekitani, J. Reeder et al., "An ultralightweight design for imperceptible plastic electronics," Nature, vol. 499, no. 7459, pp. 458-463, 2013.

[20] C. Dagdeviren, Y. Su, P. Joe et al., "Conformable amplified lead zirconate titanate sensors with enhanced piezoelectric response for cutaneous pressure monitoring," Nature Communications, vol. 5, article no. 4496, 2014.

[21] Z. L. Wang and W. Wu, "Nanotechnology-enabled energy harvesting for self-powered micro-/nanosystems," Angewandte Chemie-International Edition, vol. 51, no. 47, pp. 11700-11721, 2012.

[22] C. Dagdeviren, B. D. Yang, Y. Su et al., "Conformal piezoelectric energy harvesting and storage from motions of the heart, lung, and diaphragm," Proceedings of the National Academy of Sciences of the United States of America, vol. 111, no. 5, pp. 1927-1932, 2014.

[23] L. Francioso, C. De Pascali, I. Farella, C. Martucci, P. Cretì, and P. Siciliano, "Polyimide/PDMS flexible thermoelectric generator for ambient assisted living applications," in Proceedings of the Smart Sensors, Actuators, and MEMS V, vol. 8066, Prague, Czech Republic, April 2011.

[24] B.-H. Jo, L. M. Van Lerberghe, K. M. Motsegood, and D. J. Beebe, "Three-dimensional micro-channel fabrication in polydimethylsiloxane (PDMS) elastomer," Journal of Microelectromechanical Systems, vol. 9, no. 1, pp. 76-81, 2000.

[25] C. Luo, Q. Fu, H. Li et al., "PDMS microfludic device for optical detection of protein immunoassay using gold nanoparticles," Lab on a Chip-Miniaturisation for Chemistry and Biology, vol. 5, no. 7, pp. 726-729, 2005.

[26] M. G. King, J. A. Baragwanath, C. M. Rosamond et al., "Porous PDMS force sensitive resistors," Procedia Chemistry, vol. 1, no. 1, pp. 568-571, 2009.

[27] H.-C. Jung, J.-H. Moon, D.-H. Baek et al., "CNT/PDMS composite flexible dry electrodesfor long-term ECG monitoring," IEEE Transactions on Biomedical Engineering, vol. 59, no. 5, pp. 1472-1479, 2012.

[28] P. Yi, R. A. Awang, W. S. T. Rowe, K. Kalantar-Zadeh, and K. Khoshmanesh, "PDMS nanocomposites for heat transfer enhancement in microfluidic platforms," Lab on a ChipMiniaturisation for Chemistry and Biology, vol. 14, no. 17, pp. 3419-3426, 2014.

[29] X. Li, R. Zhang, W. Yu et al., "Stretchable and highly sensitive graphene-on-polymer strain sensors," Scientific Reports, vol. 2, pp. 2045-2322, 2012.

[30] D. J. Cohen, D. Mitra, K. Peterson, and M. M. Maharbiz, "A highly elastic, capacitive strain gauge based on percolating nanotube networks," Nano Letters, vol. 12, no. 4, pp. 1821-1825, 2012. 


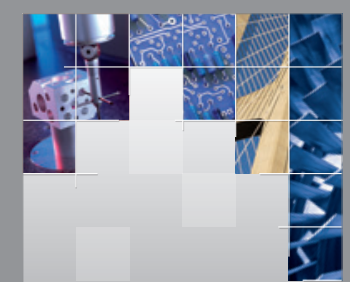

\section{Enfincering}
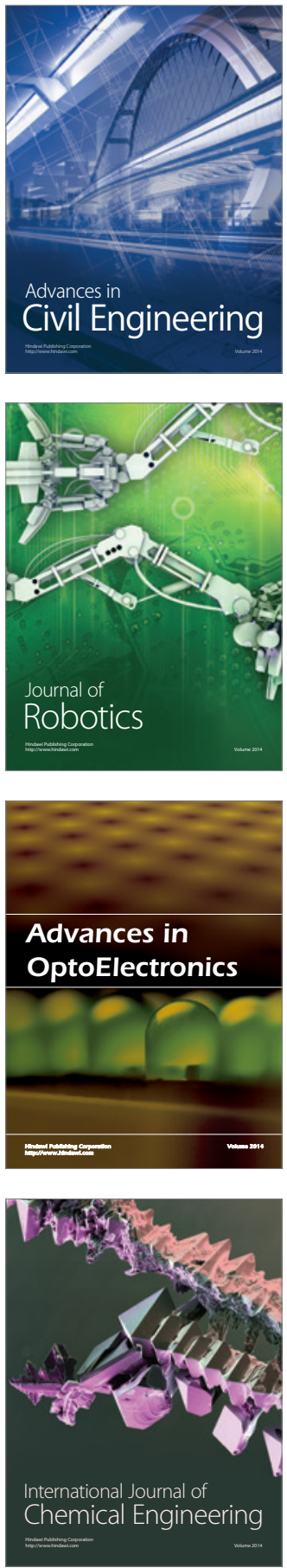

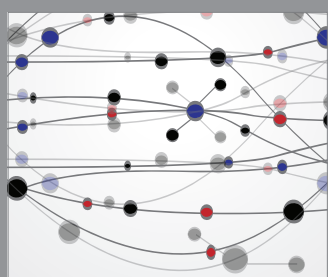

The Scientific World Journal

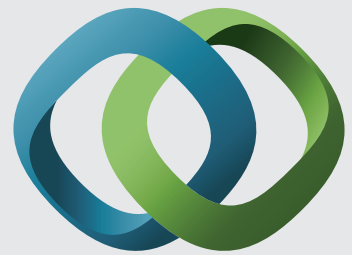

\section{Hindawi}

Submit your manuscripts at

https://www.hindawi.com
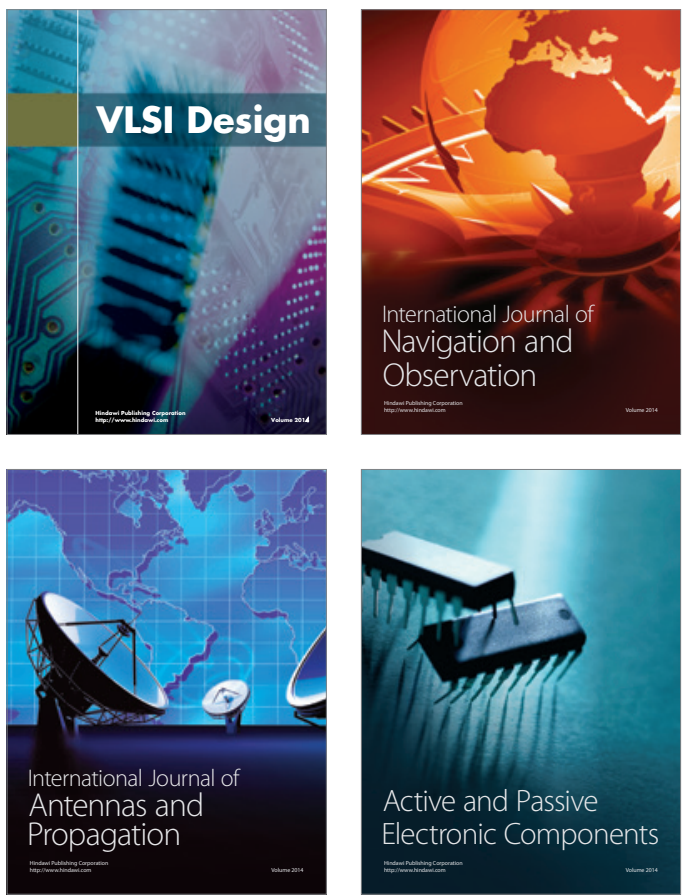
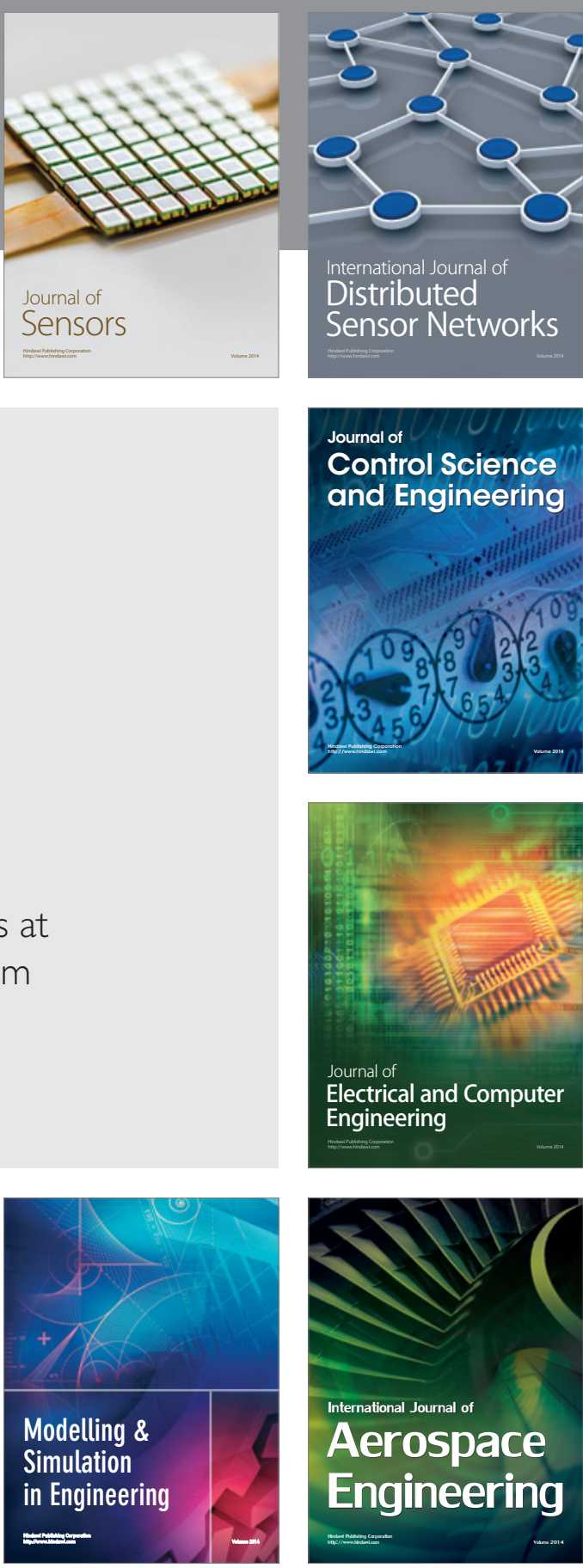

International Journal of

Distributed

Sensor Networks

$-$

Joumal of

Control Science

and Engineering
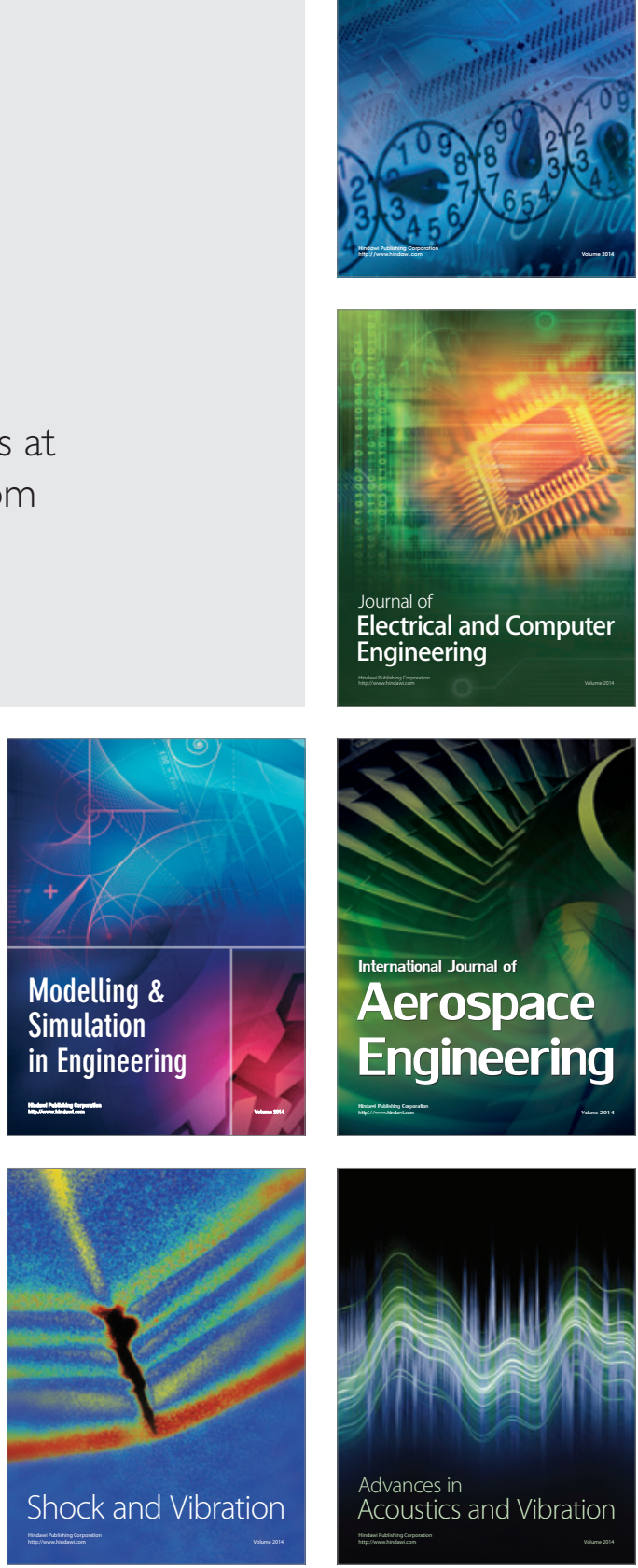